CALCULATION OF TRANSMISSION LINE IMPEDANCES

USING THE ANSYS FINITE ELEMENT PROGRAM

\author{
Marvin E. Johnson \\ June 29, 1984
}




\section{Calculation of Transmission Line Impedances Using the ANSYS Finite Element Program.}

\section{Marvin Johnson}

This paper describes numerical techniques for calculating the capacitance of an arbitrary two conductor structure. For two dimensional structures the capacitance per unit length can be easily related to the characteristic impedance of a transmission line with the same cross section. Note that this is true for lines operated below their cut off frequency, i.e., TEM mode only. Thus, one can compute the impedance of an arbitrarily shaped line. The method using the ANSYS finite element program is being used to design the high voltage transmission line for the Yale streamer chamber. This line has a transition piece between two different cross sections. It is being modeled by taking several slices throughout the transition region.

The characteristic impedance, $z_{b}$, of a loss free transmission line is

$$
Z_{0}=\sqrt{\frac{L}{C}}
$$

and the velocity, $v$, of propagation is

$$
V=\frac{1}{\sqrt{L C}}=\frac{1}{\sqrt{\mu \epsilon}}
$$

where $I$ is the inductance per unit lenqth, $C$ is the capacitance per unit length, and $\epsilon$ and $\mu$ are the permitivity and permeability of the material between the two conductors. By solving (2) for L and substituting into (1) one gets

$$
z_{0}=\frac{\sqrt{\mu \epsilon}}{C}
$$

Thus calculating the impedance is reduced to determining the capacitance per unit length. This same result is true even if there is leakage current in the dieletric; only the $\mu \in$ part will be modified. 
The ANSYS finite element program solves the heat transfer equation

$$
\nabla k \nabla T=0
$$

This is Laplace's equation which is also the solution of Maxwell's equations for static fields. By making the analogies

(S) $\mathrm{k}=\epsilon ; \mathrm{T}=\mathrm{V}$

ANSYS will solve for the electrostatic potential. Since $k$ can be different for different parts of the model, one can solve problems with multiple dieletrics.

From Gauss's law

$$
Q=\int \epsilon \vec{E} \cdot \vec{n} d S=\int \epsilon \vec{\nabla} v \cdot \vec{n} d S
$$

where $\vec{E}$ is the electric field vector, $\vec{n}$ is a unit vector normal to the surface, $V$ is the electrostatic potential and $Q$ is the charge per unit length. Since $C=Q / V$, all that is needed is to integrate the flux over a surface surrounding one of the conductors. ANSYS calculates the heat flow (i.e., the flux) into every node. By summing the heat flows into the nodes at the boundary of one of the conductors, one gets the capacitance per unit length (it is best to use the nodes at the boundary because heat flow is calculated by subtraction two potentials; at the boundary, only one of the potentials is calculated). Several examples of this method are given below.

Fig. 1 shows a quarter section of a coaxial transmission line. The analytic calculation of the impedance gives

A. Single coaxial line (See also Fig. 23).

(7) $z_{0}=96.566 \mathrm{ohms}$

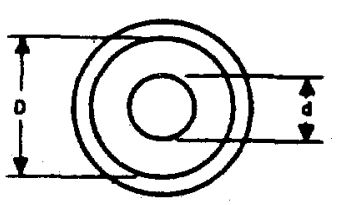

$$
\begin{aligned}
Z_{0} & =\left(138 / \mathrm{e}^{1 / 2}\right) \log _{10}(D / d) \\
& =\left(60 / \mathrm{e}^{1 / 2}\right) \log _{\mathrm{e}}(D / d) \\
\epsilon & =\text { dielectric constant } \\
& =1 \text { in air }
\end{aligned}
$$


Summing the heat flows, multiplying by 4 and dividing by $V \quad(=1$ volt) gives $\mathrm{Z}_{0}=96.50$ ohms which is within 0.18 of the analytical result. Fig. 2 shows the computed equipotentials and fig. 3 shows the input commands to generate and run the model. This problem took? seconds on the Cyber.

Figs 4 to 6 show an off center coax cable. The analytic formula gives

$U$. Eccentric line

$$
z_{0}=174.07 \text { ohms }
$$

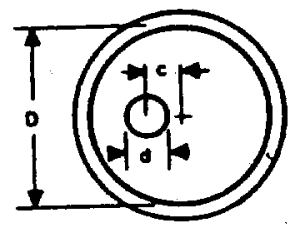

$$
\begin{aligned}
& Z_{0}=\left(60 / \epsilon^{1 / 2}\right) \cosh ^{-1} U \\
& U=\frac{1}{2}\left[(D / d)+(d / D)-\left(4 c^{2} / d D\right)\right]
\end{aligned}
$$

The ANSYS result is 173.84 ohms which differs by $0.13 \%$

Figs 7 to 9 show a twinax cable with sheath return. That is, the two center conductors are at the same potential. The analytic solution is

$L$. Wires in parallel-sheath return

$$
z_{0}=96.41 \text { ohms }
$$

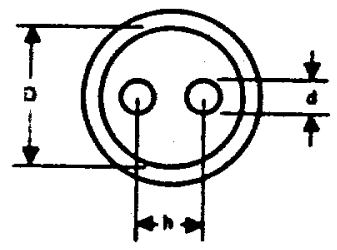

$$
\begin{aligned}
& \text { For } d \ll D, h, \\
& \qquad \begin{aligned}
Z_{0} & =\left(69 / \epsilon^{1 / 2}\right) \log _{10}\left[\left(\nu / 2 \sigma^{2}\right)\left(1-\sigma^{4}\right)\right] \\
\sigma & =h / D \\
\nu & =h / d
\end{aligned}
\end{aligned}
$$

and the ANSYS result is 94.66 ohms. This result differs by 1.88 . Note, however, that the analytic solution is an approximation.

The above method uses derived quantities to calculate the capacitance. A method that uses the nodal values directly would be more accurate. Such a method is described below [1] 
The finite element method for Laplace's equation minimizes the total energy of a system. The capacitance is related to the total energy by

$$
w=\frac{1}{2} C V_{0}^{2}
$$

where $V_{\text {. }}$ is the potential difference between the two conductors. This method finds the nodal values which minimize the total energy and then uses these values to calculate the total energy of the system. Substituting this into (8) gives the capacitance.

The relationship between the normal finite element matrices and the total energy of the system is particularly simple. This relationship is derived below. In two dimensions the total energy of a system is

$$
\begin{aligned}
w & =\frac{1}{2} \int \vec{E} \cdot \epsilon \vec{E} d \Omega=\frac{1}{2} \int \overrightarrow{\nabla v} \cdot \epsilon \overrightarrow{\nabla v} d \Omega \\
& =\frac{1}{2} \int \epsilon\left[\left(\frac{\partial v}{\partial x}\right)^{2}+\left(\frac{\partial v}{\partial y}\right)^{1}\right] d \Omega
\end{aligned}
$$

where $\vec{E}$ is the electric field vector and $E$ is the permitivity of the material. After an object is broken up into finite elements (see fig. 1), the total energy can be expressed as a sum of the energies of the individual elements.

$$
w=\frac{1}{2} \sum_{e} \int_{E L E M E N T} E\left[\left(\frac{\partial v}{\partial x}\right)^{2}+\left(\frac{\partial v}{\partial y}\right)^{2}\right] d \Omega_{e}
$$

where the integral is now over the volume of one element.

Next, one must express the value of the potential at any point inside the element in terms of the values at its nodes:

$$
V_{e}=\sum N_{i}(x, y) V_{i}
$$

Here, $V_{i}$ is the potential at node $i$ and the $N_{c}$ contain the information about the geometry of the element. Note that the sum in (11) is a sum over one element. If the element is a triangle, 
$\mathrm{TM}-1270$

5

the sum would be from 1 to 3 . The $N_{i}$ are commonly called shape functions. They can best be described by a one dimensional example. If one assumes a linear variation of potential over a line segment, then

$$
V=a x+b
$$

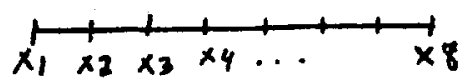

Solving for $a$ and $b$ in terms of the nodal values $X 1$ and $X 2$ (I have used nodes 1 and 2 for clarity; they could be any adjacent pair) gives

$$
a=\frac{v_{2}-v_{1}}{x_{2}-x_{1}} \quad b=\frac{v_{1} x_{2}-v_{2} x_{1}}{x_{2}-x_{1}}
$$

Rearranging gives

$$
V=\left(\frac{x-x_{2}}{x_{2}-x_{1}}\right) v_{1}+\left(\frac{x-x}{x_{2}-x_{1}}\right) v_{2}
$$

Thus, the shape functions $\mathrm{N} 1$ and $\mathrm{N} 2$ for this element are

$$
N_{1}=\left(\frac{x-x_{2}}{x_{2}-x_{1}}\right) \quad N_{2}=\left(\frac{x_{1}-x}{x_{2}-x_{1}}\right)
$$

Putting (11) into (10) gives

$$
W=\frac{1}{2} \sum_{e} \int \epsilon\left[\left(\sum_{i} \frac{\partial N_{i}}{\partial x} V_{i}\right)^{2}+\left(\sum_{i} \frac{\partial N_{i}}{\partial y} V_{i}\right)^{2}\right] d \Omega_{e}
$$

Again, the $i$ sum is only over one element.

We wish to choose the nodal values $v_{i}$ such that $W$ is a minimum. This implies that

$$
\frac{\partial W}{\partial V_{i}}=0=\sum_{e} \int \epsilon\left[\left(\frac{\partial N_{i}}{\partial x} \sum_{j} \frac{\partial N_{j}}{\partial x} v_{j}\right)+\left(\frac{\partial N_{i}}{\partial y} \sum_{j} \frac{\partial N_{j}}{\partial y} v_{j}\right)\right] d \Omega_{e}
$$


The energy of every part of a field is a minimum so the integral of each individual element is also zero. The integral in (17) for one element can be written in matrix form as:

$$
\begin{aligned}
& \int\left(\frac{\partial N_{1}}{\partial x} \frac{\partial N_{1}}{\partial x}+\frac{\partial N_{1}}{\partial y} \frac{\partial N_{1}}{\partial y}\right) V_{1} d R_{e}+\cdots+\int\left(\frac{\partial N_{1}}{\partial x} \frac{\partial N_{j}}{\partial x}+\frac{\partial N_{1}}{\partial y} \frac{\partial N_{j}}{\partial y}\right) V_{j} d \Omega_{e}=\varnothing \\
& \int\left(\frac{\partial N_{2}}{\partial x} \frac{\partial N_{1}}{\partial x}+\frac{\partial N_{2}}{\partial y} \frac{\partial N_{1}}{\partial y}\right) V_{1} d \Omega_{e}+\cdots+\int\left(\frac{\partial N_{2}}{\partial x} \frac{\partial N_{j}}{\partial x}+\frac{\partial N_{2}}{\partial y} \frac{\partial N_{j}}{\partial y}\right) V_{j} d \Omega_{e}=\varnothing
\end{aligned}
$$

$$
\int\left(\frac{\partial N_{j}}{\partial x} \frac{\partial N_{1}}{\partial x}+\frac{\partial N_{j}}{\partial y} \frac{\partial N_{1}}{\partial y}\right) V_{1} d R_{e}+\cdots+\int\left(\frac{\partial N_{j}}{\partial x} \frac{\partial N_{j}}{\partial x}+\frac{\partial N_{j}}{\partial y} \frac{\partial N_{j}}{\partial y}\right) V_{j} d \Omega_{\varepsilon}=\varnothing
$$

or

$$
\left[k_{e}\right]\left[v_{e}\right]=[0]
$$

where

$$
k_{i j}=\int\left(\frac{\partial N_{i}}{\partial x} \frac{\partial N_{j}}{\partial x}+\frac{\partial N_{i}}{\partial y} \frac{\partial N_{j}}{\partial y}\right) d \Omega_{e}
$$

The sum over all elements can be written as

$$
[K][V]=[0]
$$

Unless all of the $\mathrm{V}_{i}$ on the boundary are zero, (20) has a non zero solution for the vector [V].

Next compute the energy. If we go back to (16) and expand the integral, we $g \in t$ 


$$
\begin{gathered}
W=\frac{1}{j} \int \epsilon\left[\frac{\partial N_{1}}{\partial x} v_{1} \frac{\partial N_{1}}{\partial x} v_{1}+\frac{\partial N_{1}}{\partial y} v_{1} \frac{\partial N_{1}}{\partial y} v_{1}\right] d \Omega_{e}+\cdots+ \\
\int \epsilon\left[\frac{\partial N_{1}}{\partial x} v_{1} \frac{\partial N_{j}}{\partial x} v_{j}+\frac{\partial N_{1}}{\partial y} v_{1} \frac{\partial N_{j}}{\partial y} v_{j}\right] d \Omega_{e}+\cdots+ \\
\int\left[\epsilon \frac{\partial N_{j}}{\partial x} v_{j} \frac{\partial N_{j}}{\partial x} v_{j}+\frac{\partial N_{j}}{\partial y} v_{j} \frac{\partial N_{j}}{\partial y} v_{j}\right] d \Omega_{e}
\end{gathered}
$$

or in terms of the global matrices

$$
W=\frac{1}{2} \sum_{e} \in\left[V_{e}\right]^{T}\left[K_{e}\right]\left[V_{e}\right]=\frac{1}{2} \in[V]^{T}[k][V]
$$

where $[K]$ is the usual finite element matrix. One need only compute the normal finite element solution of Laplace's equation keeping the original $[K]$ matrix. Then one computes (22) and substitutes $W$ into $(8)$ to get the capacitance. Note that this method is not dependent on the detailed structure of the $\mathrm{N}_{C} ;$ any element formulation will work. The above analysis can easily be extended to 3 dimensions.

This method is not yet available in ANSYS but could presumably be made available if the need arose. Since the first method works quite well, it may not be needed.

[1]Daly and Helps,Electronics Letters, [8],5, (1972), pl32. 


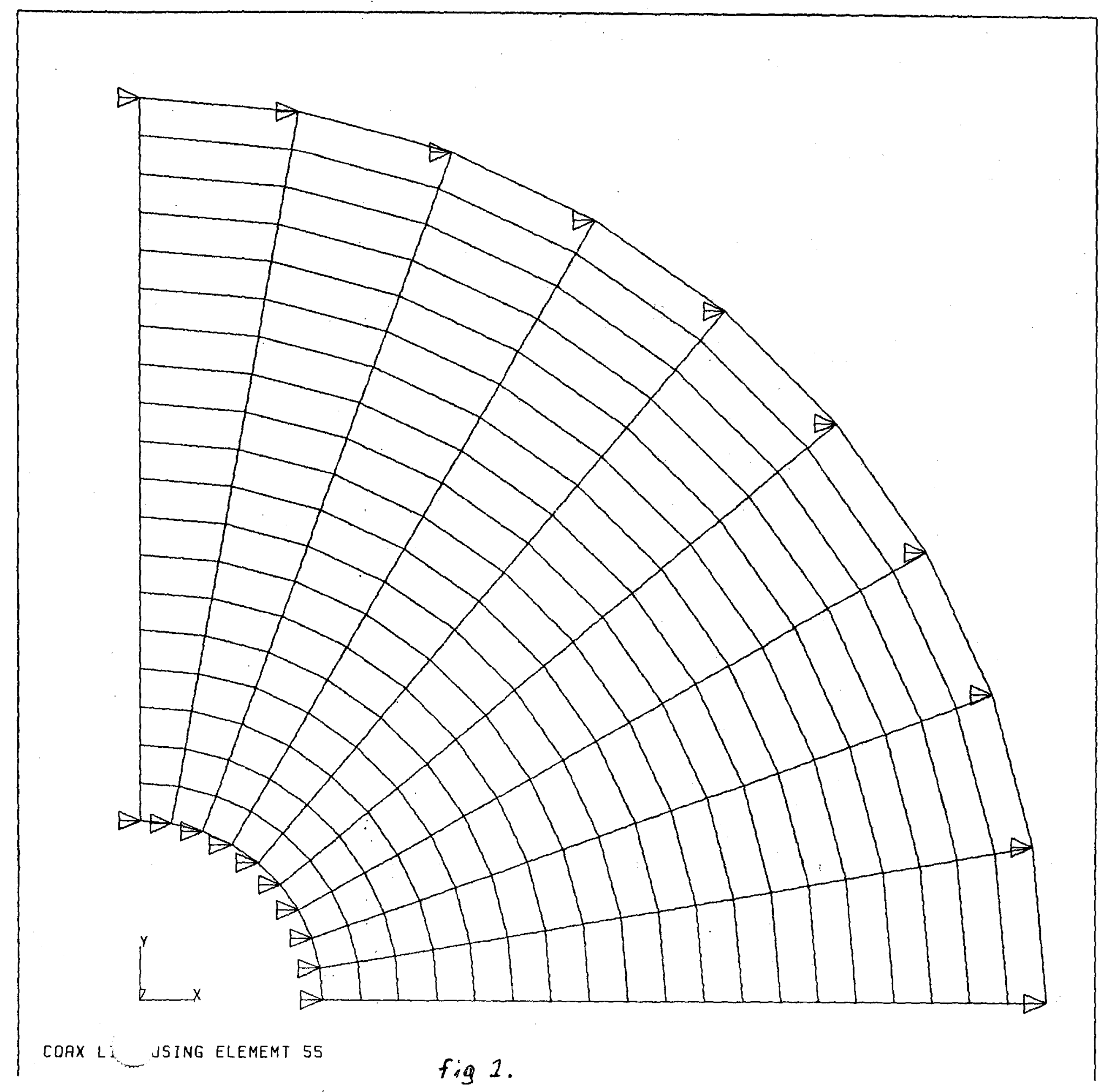

ANSYS

84/6/28

10.8881

PLOT NO. 3

PREP7 ELEMENTS

$N T B C=1$

ORIG SCALING

$Z V=1$

OIST $=.297$

$X F=.23$

$Y F=.25$ 


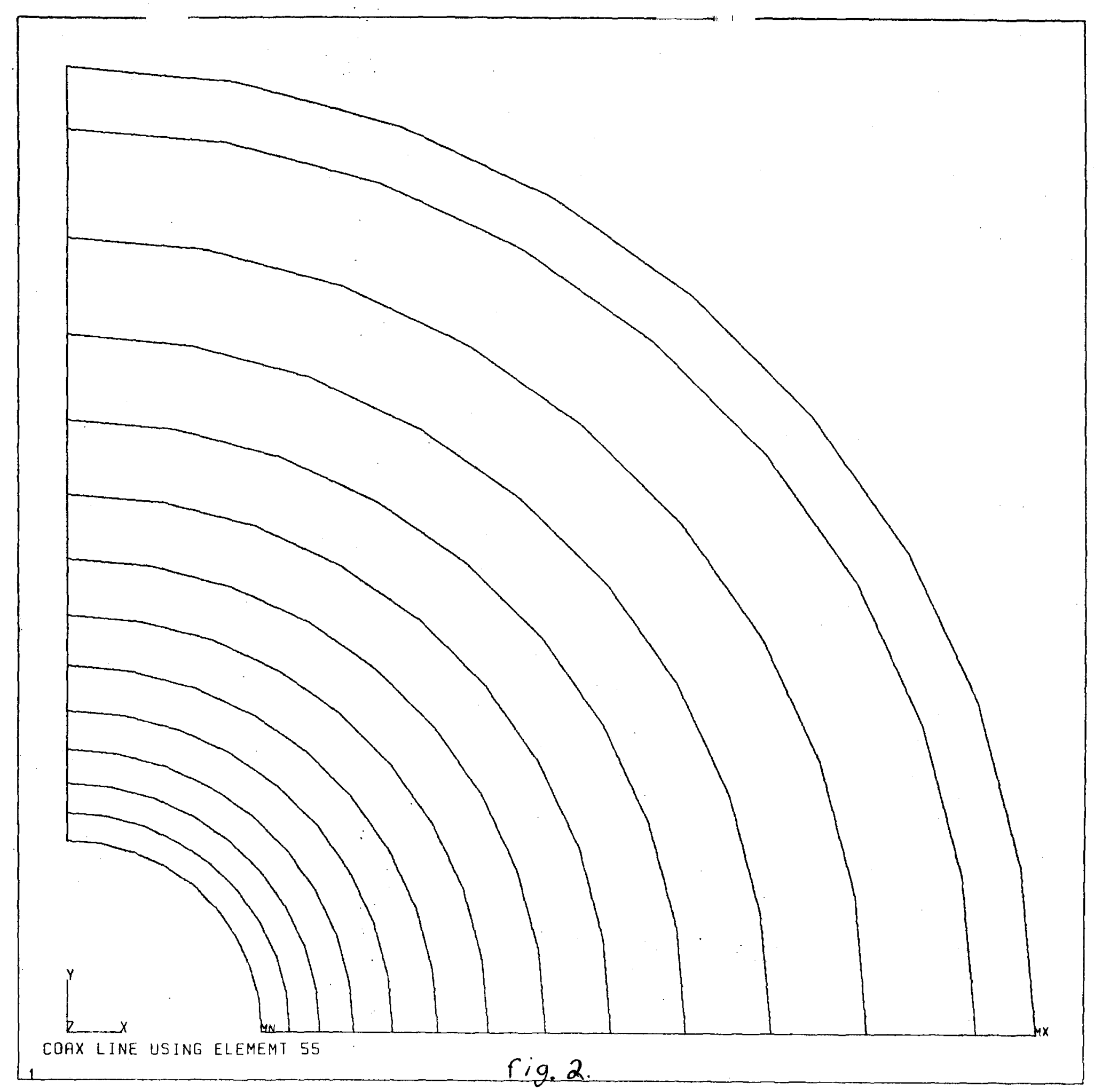

ANSYS

$84 / 6 / 28$

11.0919

PLOI NO.

POST1

STEP $=1$

I TER $=1$

STRESS PLOT

TEMP

ORIG SCALING

$Z V=1$

DIST $=.275$

$X F=.25$

$Y F=.25$

EDGE

$M X=1$

$M N=0$

INC $=.08$ 


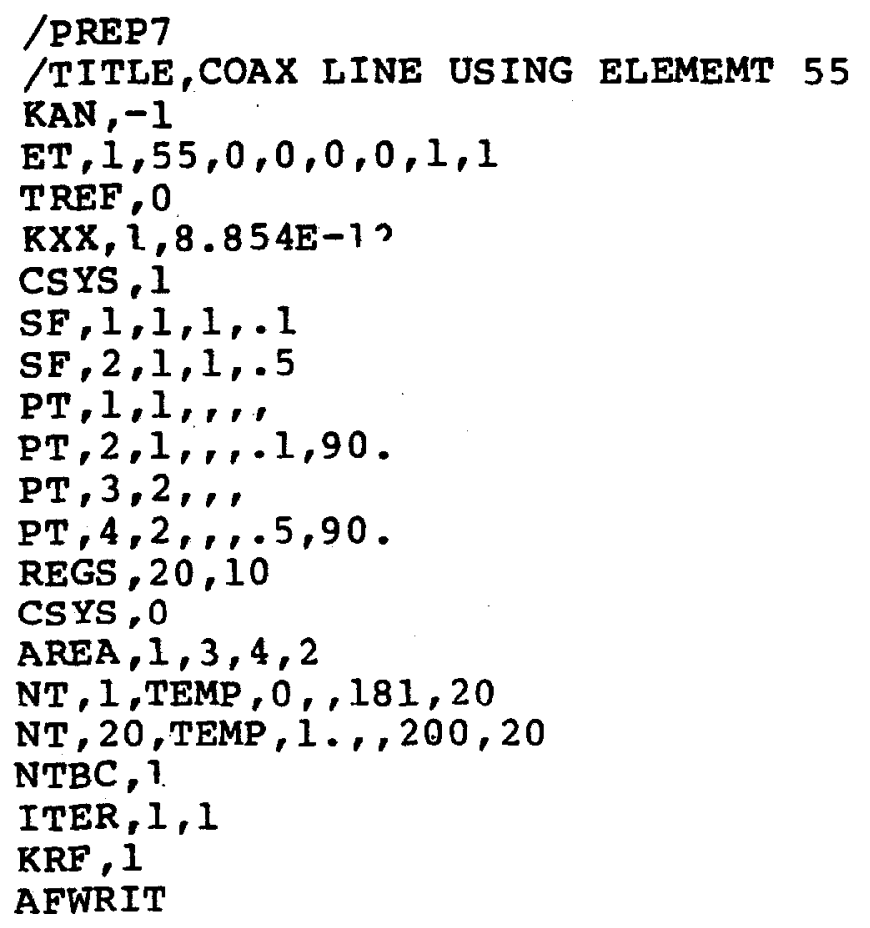

The following commands use the post processor to sum the charge around the center conductor

/POSTI

$C * \star \star$ FOR OFFSET CABLE

$C \star \star \star$ VOLTS $=1.0 ! ! ! ! ! !$

SET

NRSEL , NODE , $25,500,25$

NASEL, NODE , 525,975,25

PRRFOR

NALL

Fig. 3 Input file for Coax Cable 


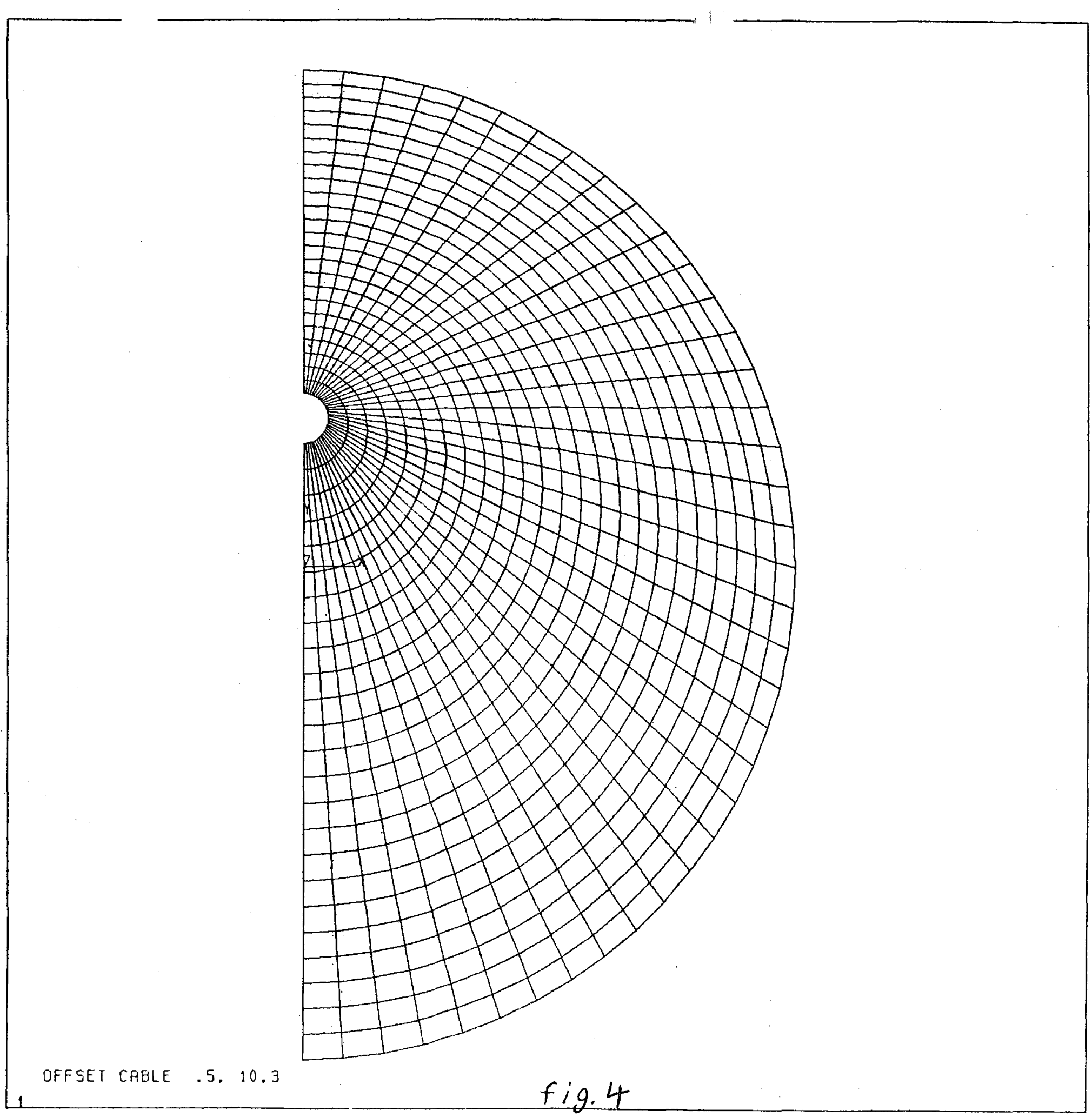

ANSYS

$84 / 6 / 27$

13.0222

PLOT NO. 2

PREP7 ELEMENTS

ORIG SCALING

$2 V=1$

$D I S T=11$

$X F=5$ 

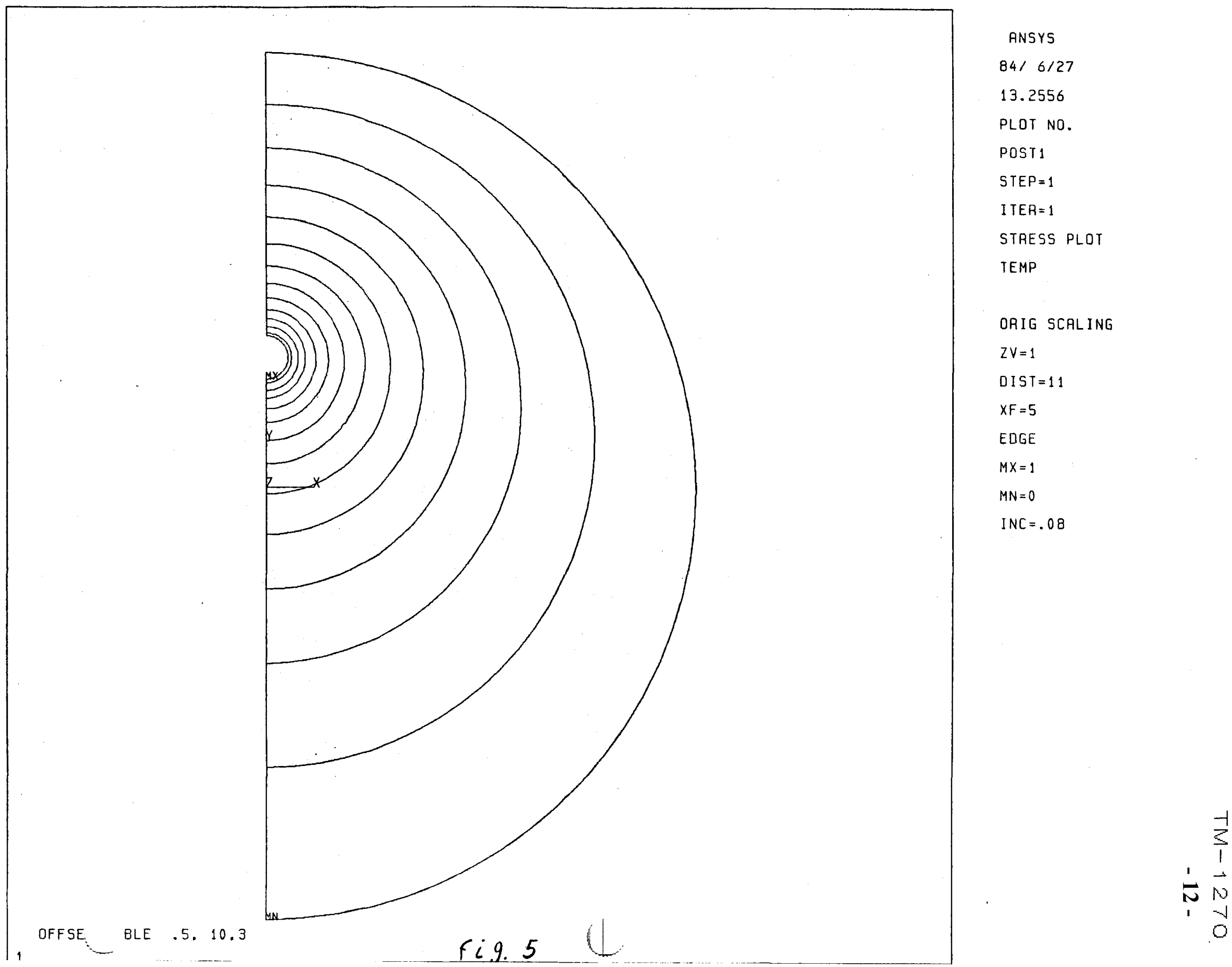


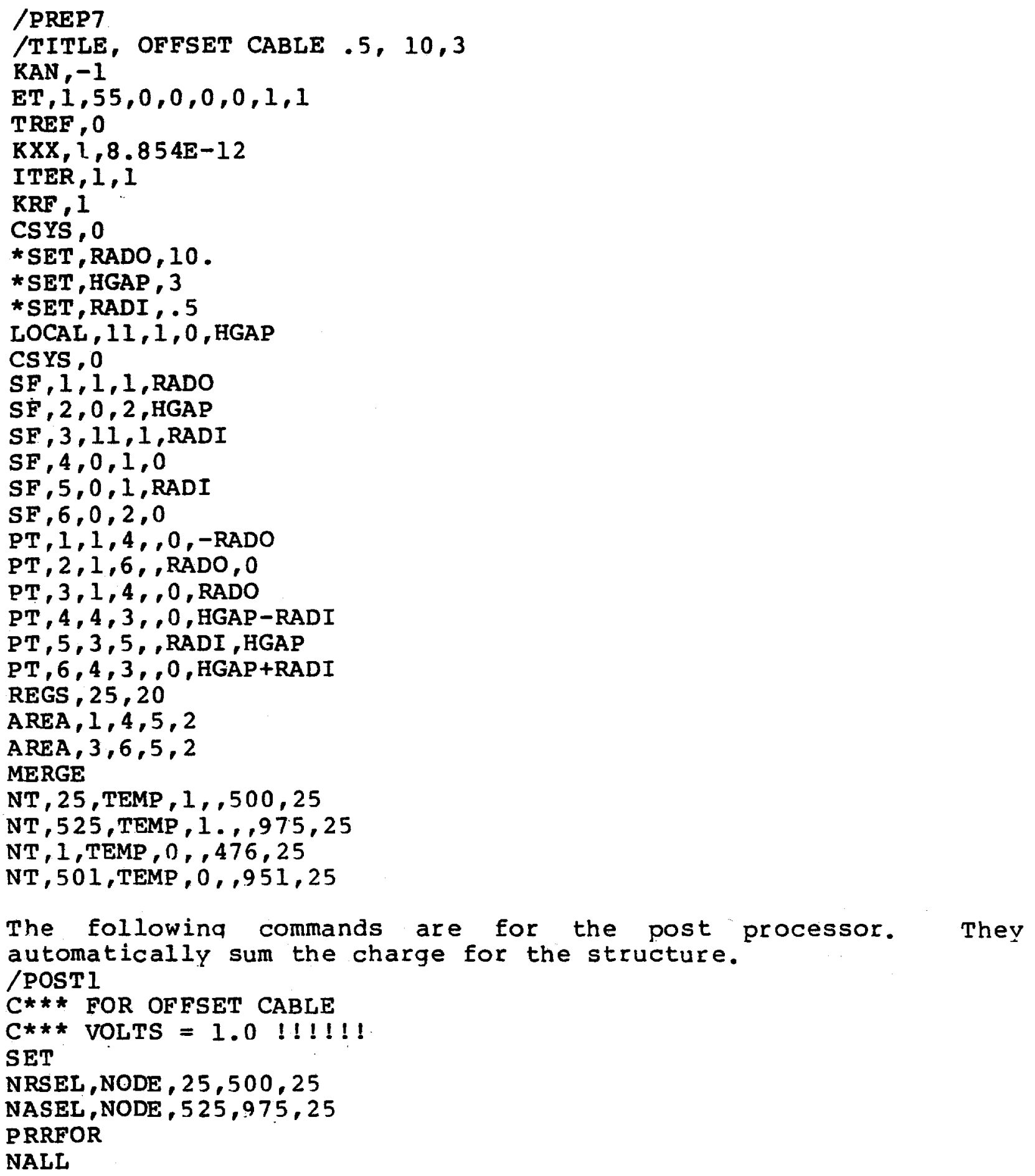

Fig. 6 Commands for an off center Coax cable 


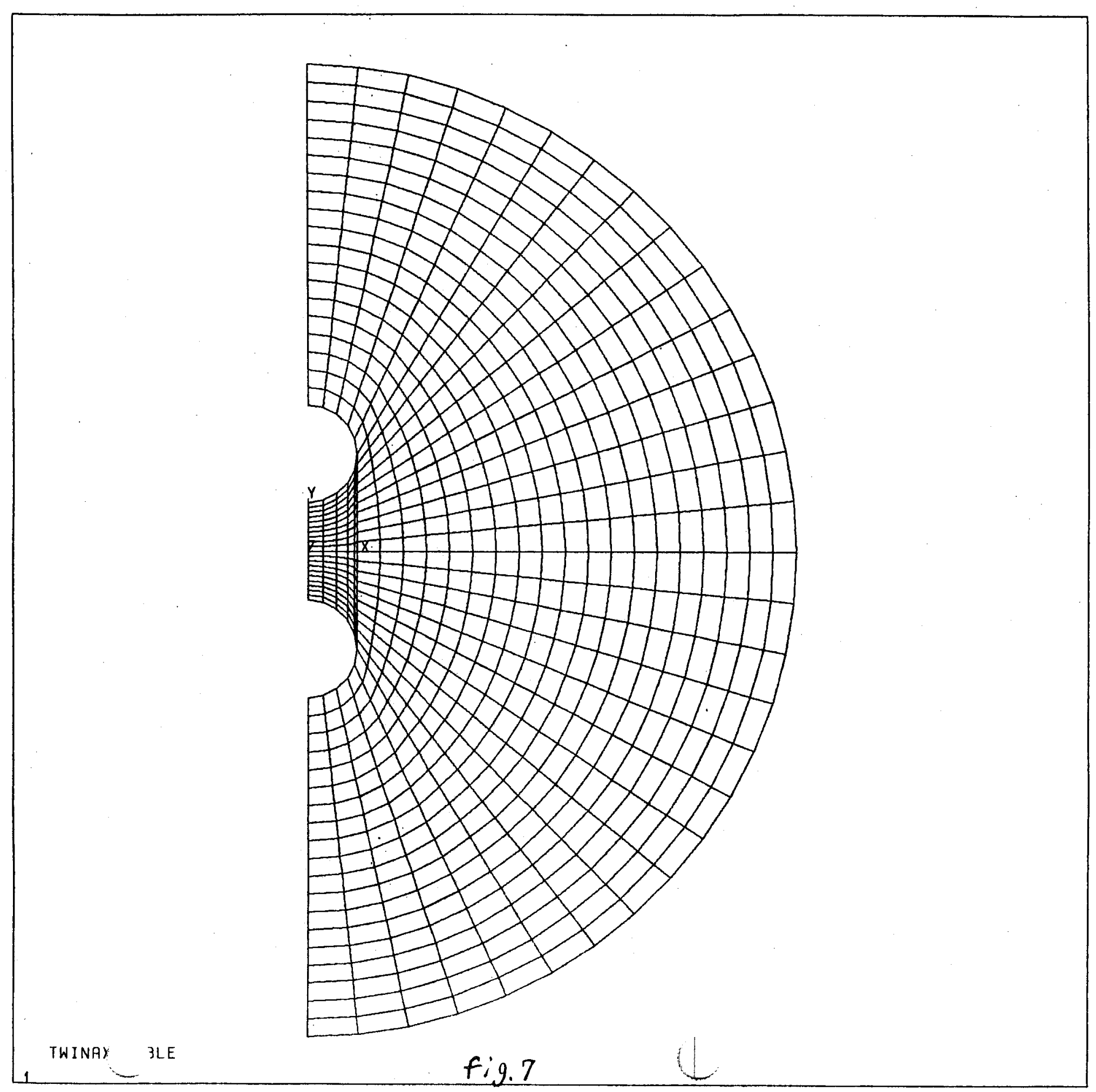

\section{ANSYS}

$84 / 6 / 27$

22.1036

PLOT NO. 2

PREP7 ELEMENTS

ORIG SCALING

$Z V=1$

DIST $=11$

$X F=5$ 


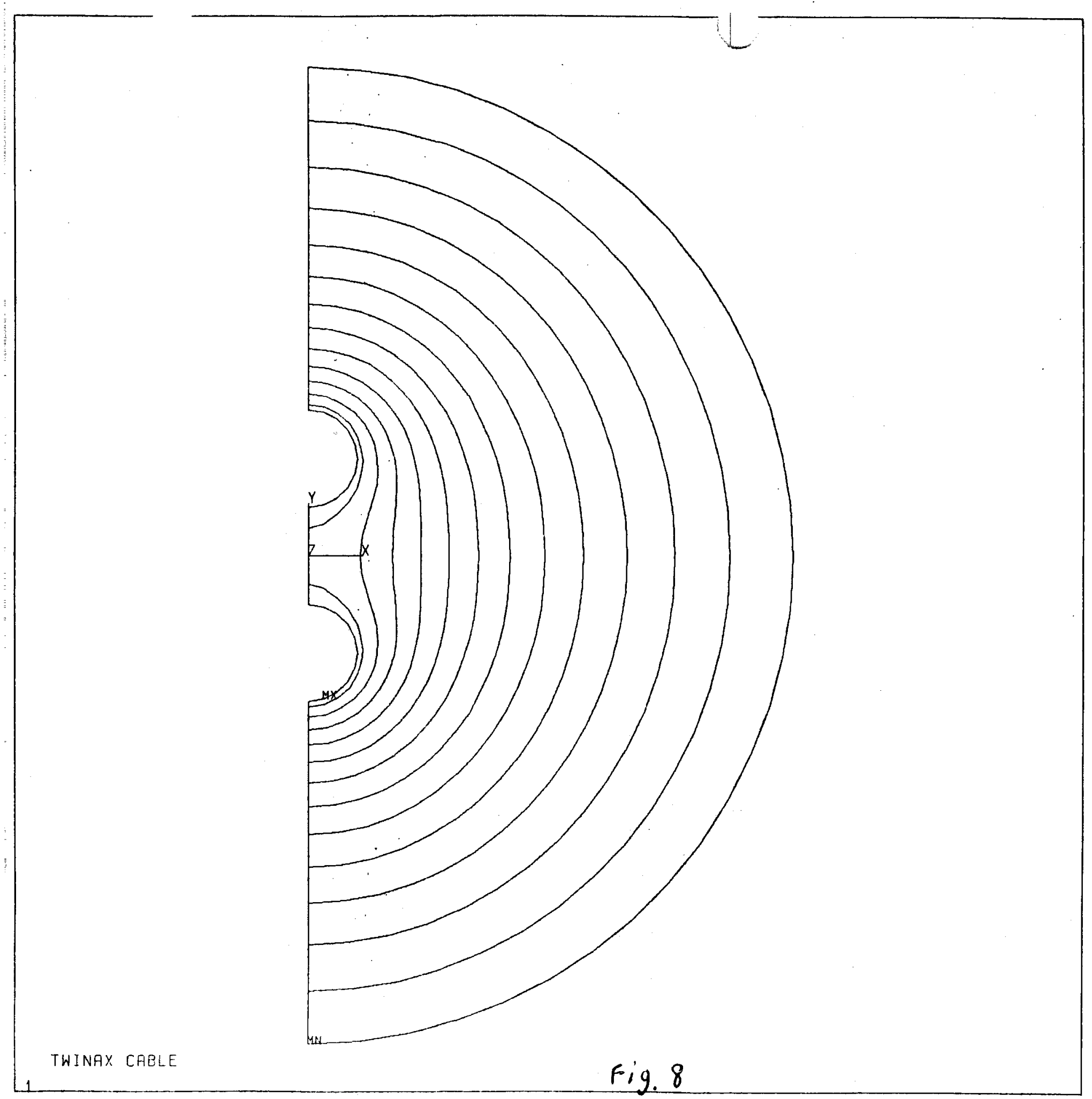

\section{ANSYS}

$84 / 6 / 27$

22.3072

PLOT NO.

POST1

STEP $=1$

I TER $=1$

STRESS PLOT

TEMP

ORIG SCAL ING

$Z V=1$

DIST $=11$

$X F=5$

EDGE

$M X=1$

$M N=0$

$I N C=.08$ 


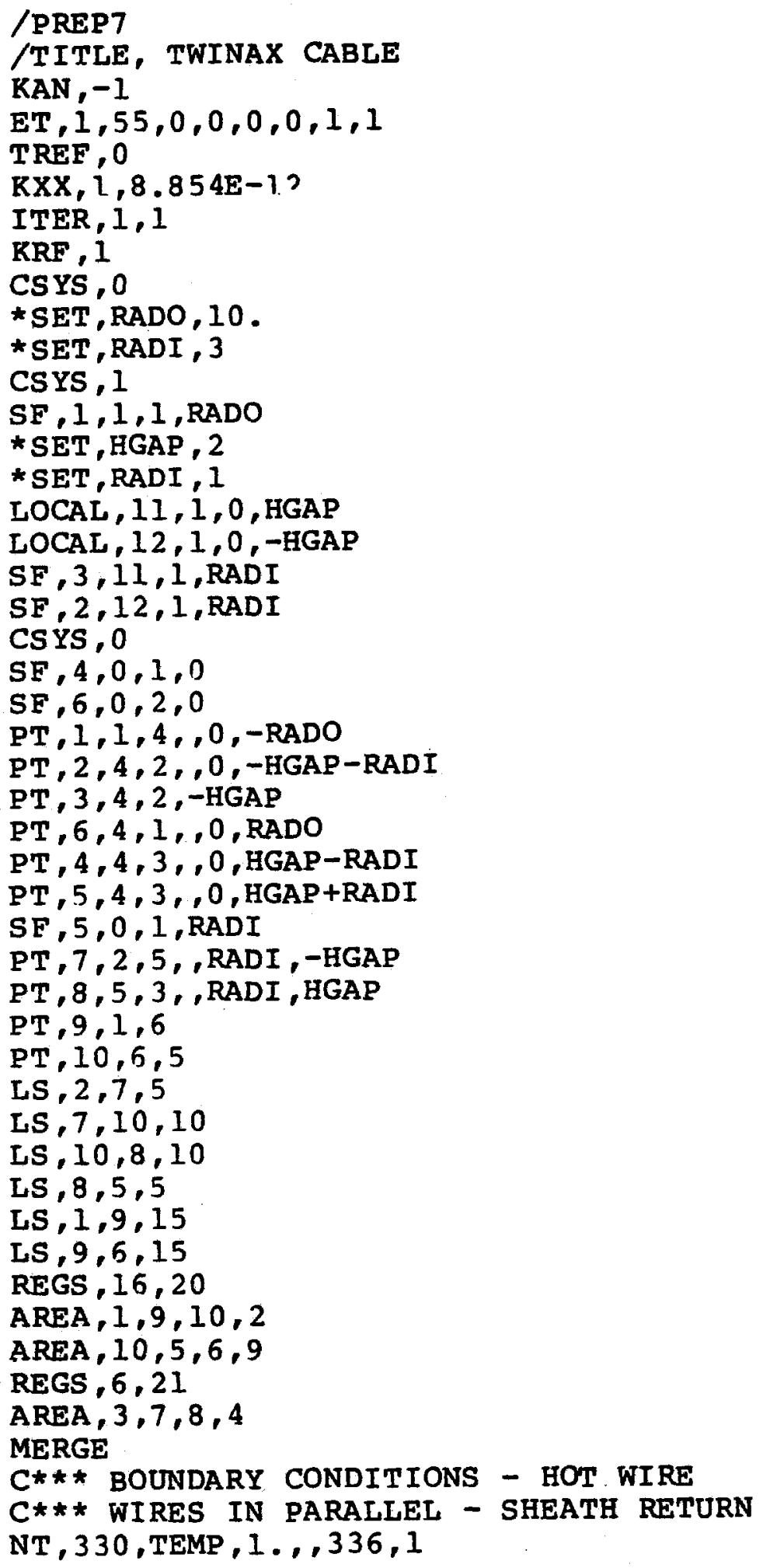


NT, 761, TEMP , 1, , 765, 1

NT, 641,TEMP, $1 \ldots, 645,1$

NT, 305, TEMP, $1, \ldots, 311,1$

NT, 626,TEMP $, 0.00,, 640,1$

NT, 1,TEMP , 0.00, 16,1

The following commands are for the post processor. They sum the computed charge.

\section{/POST1}

$C * \star *$ FOR TWINAX CABLE

$C * \star *$ WIRES IN PARALLEL - SHEATH RETURN

SET

NRSEL, NODE , 761,765,1

NASEL, NODE, $330,336,1$

NASEL, NODE , 305, 311, 1

NASEL, NODE , 641,645,1

PRRFOR

NALL

Fig 9 Commands to generate the twinax cable model. Note that the two inner conductors are at the same potential. 